This item was submitted to Loughborough's Research Repository by the author.

Items in Figshare are protected by copyright, with all rights reserved, unless otherwise indicated.

\title{
Friction in ultra-thin conjunction of valve seals of pressurised metered dose inhalers
}

PLEASE CITE THE PUBLISHED VERSION

http://dx.doi.org/10.1016/j.wear.2009.12.003

PUBLISHER

(C) Elsevier

VERSION

AM (Accepted Manuscript)

LICENCE

CC BY-NC-ND 4.0

\section{REPOSITORY RECORD}

Prokopovich, Polina, Stephanos Theodossiades, Homer Rahnejat, and D. Hodson. 2019. "Friction in Ultra-thin Conjunction of Valve Seals of Pressurised Metered Dose Inhalers". figshare.

https://hdl.handle.net/2134/13330. 
This item was submitted to Loughborough's Institutional Repository (https://dspace.lboro.ac.uk/) by the author and is made available under the following Creative Commons Licence conditions.

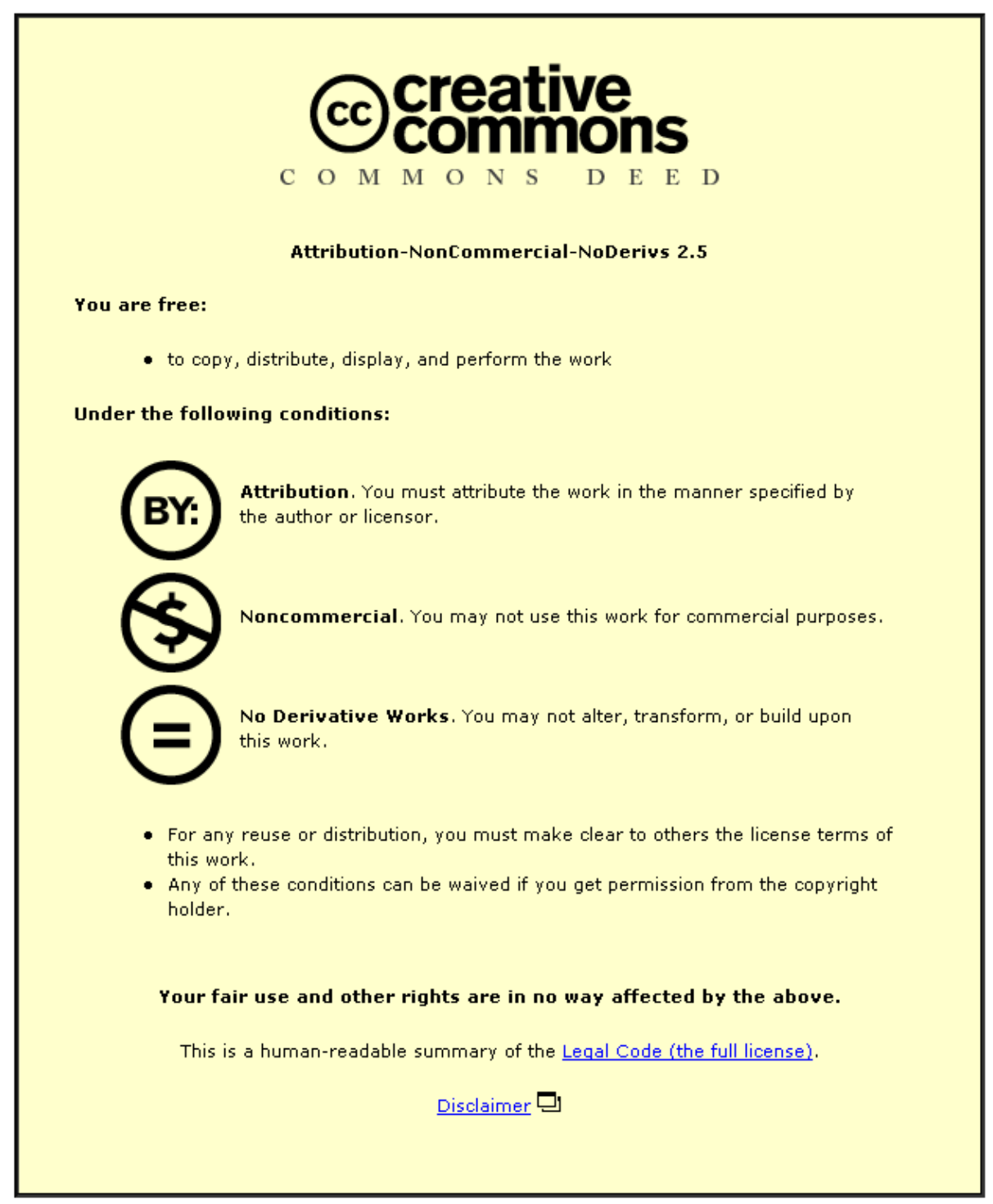

For the full text of this licence, please go to: http://creativecommons.org/licenses/by-nc-nd/2.5/ 


\section{Friction in Ultra-thin conjunction of Valve Seals of}

\section{Pressurised Metered Dose Inhalers}

3

4

P. Prokopovich ${ }^{1}$, S. Theodossiades ${ }^{1}$, H. Rahnejat ${ }^{1, *}$ and D. Hodson ${ }^{2}$

5

6

$7{ }^{1}$ Wolfson School of Mechanical \& Manufacturing Engineering, Loughborough University,

8 Loughborough, LE11 4NX, UK

$9 \quad{ }^{2}$ AstraZeneca R\&D Charnwood, Bakewell Road, Loughborough, LE11 5RH, UK

* Corresponding author:

Email: h.rahnejat@lboro.ac.uk

Tel : +44 (0)1509 227569

Fax: +44 (0)1509 227569

15

16 Wolfson School of Mechanical \& Manufacturing Engineering

17 Loughborough University

18 Loughborough

19

LE11 4NX, UK

20 
Abstract

In many drug dispensing devices, such as syringes and inhalers, a rubber disk is used as a seal. During device actuation the seal is subjected to friction which in turn causes its deformation. This can lead to suboptimal performance of the device and consequent variability in delivered dose. Seal friction is complex, arising from adhesion of rubber in contact with the moving interface, viscous action of a thin film of fluid and deformation of seal asperities. Therefore, the first step in understanding the conjunctional behaviour of rubber seals is the fundamental study of mechanisms of friction generation. A developed model can then be validated against measurements. The validated model can then be used to predict product performance, robustness and variability due to manufacturing tolerances.

A friction model, based on the aforementioned mechanisms, for prediction of seal friction has been developed and validated against measured friction tests performed on both nano and component level scales. Pressure changes in the metering chamber have been taken into account in the model. Friction data are presented for nitrile rubber, using a silicon nitride AFM tip for nano-scale interactions and polybutylene terephthalate (PBT) for asperity interactions at a component level, where a traditional friction test apparatus is utilised.

Reasonable agreement is found between measurements and model predictions for the nanoscale coefficient of friction of rubber against silicon nitride. Similarly, good agreement has been obtained for the mean coefficient of friction of rubber against PBT. It was found that the model was capable of predicting static friction coefficient reasonably well and the contribution to the coefficient of friction was mostly due to adhesive friction. The inputs of viscous and ploughing friction were negligible.

Keywords: pMDI valve, elastomeric seals, friction, adhesion. 
49 Nomenclature:

50 A Real contact area

$51 \quad A_{i} \quad$ Asperity contact area

$52 \quad A_{c} \quad$ Area under the curve of friction force versus pressure

$53 \quad a \quad$ Hertzian contact half-width

$54 \quad b \quad$ Undeformed cross-sectional seal diameter

$55 \quad D_{f} \quad$ Fractal dimension

$56 \quad D_{s} \quad$ Diameter of the sealed element

$57 \quad$ DF $\quad$ Degree of freedom

$58 \quad E \quad$ Reduced (effective) elastic modulus of the contacting pair

$59 \quad E_{r u b} \quad$ Elastic modulus of the nitrile rubber

$60 E_{\text {stem }} \quad$ Elastic modulus of the stem

$61 \quad F_{a} \quad$ Adhesive friction force

$62 \quad F_{d} \quad$ Ploughing friction force

$63 \quad F_{v} \quad$ Viscous friction force

$64 \quad F_{f}^{a v} \quad$ The average total friction force

$65 \quad f_{d} \quad$ Single asperity ploughing friction force

$66 \quad G \quad$ Scaling constant

$67 \quad g \quad$ Deformed cross-sectional seal diameter

$68 \quad h \quad$ Film thickness

$69 \quad l \quad$ Base diameter of a hemispherical asperity

$70 \quad m \quad$ Equivalent mass of an asperity in sliding motion

$71 \quad N \quad$ Number of asperities 
$72 \quad P w \quad$ Power

$73 p \quad$ Chamber pressure

$74 \Delta p \quad$ Pressure difference

$75 p_{\text {cont }}$ Contact pressure

$76 \quad p_{m} \quad$ Maximum Hertzian pressure

$77 \quad p$-value Probability

$78 \quad R_{c} \quad$ Increased radius of conforming contact

$79 \quad R^{\prime} \quad$ Radius of a hemispherical-shaped asperity

$80 \quad R_{q} \quad$ RMS surface roughness

$81 R_{q}^{\text {comp }}$ RMS composite surface roughness

$82 \quad r_{a} \quad$ Radius of a typical asperity

83 RSS Residual sum of squares

$84 u \quad$ Speed of entraining motion of fluid into the contact area

$85 \quad V \quad$ Relative sliding velocity of the valve

$86 \quad W \quad$ Applied normal load

$87 \quad W_{i} \quad$ Normal load on a hemispherical asperity

$88 \quad w \quad$ Effective width of the contact seal-stem

$89 \quad z_{0} \quad$ Height of asperity in ploughing action

$90 \quad \alpha \quad$ Calibration factor

$91 \quad \beta \quad$ Proportion of kinetic energy causing ploughing

$92 \quad \delta_{i} \quad$ Deflection for an asperity

$93 \quad \varepsilon \quad$ Squeeze ratio

$94 \quad \eta \quad$ Dynamic viscosity of the fluid 
$95 v_{r u b} \quad$ Poisson's ratio of the nitrile rubber

$96 \quad \tau_{s} \quad$ Average shear strength of the dry contact

$97 \Phi \quad$ Function in ploughing friction

$98 \psi \quad$ Proportion of the contact in direct surface interactions

99 


\section{Introduction}

In recent years several significant changes have taken place in pressurised metered dose inhaler (pMDI) valves. These include the replacement of CFC (chlorofluorocarbon) propellants, deemed to be damaging to the ozone layer with HFA (hydrofluoroalkane) based propellants, as well as the introduction of new elastomers for the containing valve seals. This transition has provided an opportunity to re-evaluate inhaler performance as noted by Everard [1]. The valve in the pMDI is one of the key integral components for device performance. This was noted in the studies carried out in the transition from CFC to HFA by Schultz [2].

In many inhalation devices, an elastomeric material, usually a form of rubber is placed around a moving stem to seal the formulation within a chamber. During the movement of the stem the seal is subjected to friction, increasing the deformation of the sealing area. This deformation contributes towards the perceived challenges relating to valve leakage, drug adsorption, dose variability changes, "loss of dose" and " loss of prime” effects [3]. Therefore, the tribological behaviour plays an important role when considering new propellants with active compounds.

The tribological behaviour in a medical device is influenced by dimensional tolerance of the moulded or cut components, as well as by the rheological characteristics of any fluid or propellant-drug-surfactant mixture, referred to as the formulation. The assessment of seal friction within the device is crucial in evaluating its design space. Material characteristics are capable of being measured on a nano-scale possibly as part of the in-line manufacturing process or as a quality check. In-situ measurement of these characteristics proves problematic. The benefit of linking the nano-scale to component-scale provides a possible 
route to the assessment of dimensional or material properties that need to be controlled in order to guarantee the reliability, robustness and manufacturability of the delivery device.

When the seal is fitted into the device it undergoes some global deformation, which alters the shape of its conjunction with the contacting sliding stem, whose motion actuates the inhaler valve. Therefore, there is a corresponding tensile force which strives to return the seal to its undeformed state. The shape of the ring is also affected by the canister pressure, which compresses the seal, with a corresponding outward reaction. These resistive forces are balanced by the contact force between the seal and the stem, which is as the result of any generated fluid pressure in the conjunction and asperity pressures. During the device actuation (motion of stem) the seal is subjected to friction, which is generated by viscous action of a very thin adsorbed film on the contiguous surfaces and asperity-pair interactions as described by Grimble et al [5].

Friction is quite complex, arising from adhesion and viscous action of a thin film. Therefore, the first step in understanding the conjunctional behaviour of such elastomeric seals is the fundamental study of mechanisms of friction generation. A developed model can then be validated against measurements, prior to its use in a multi-body dynamic model [6] of the inhaler valve to predict product performance, robustness and variability due to manufacturing tolerances. This paper undertakes two distinct studies.

Firstly, a friction model for the rough elastomeric material, typically used for valve seals is developed. The model is then validated against measurements at nano-scale. Friction data is presented for nitrile rubber, using a silicon nitride AFM tip for nano-scale interactions. The validation is then extended to macro-scale motion of an instrumented trolley, incorporating 
an elastomeric surface sliding on a polymeric counterface. Therefore, these tests carried out for polybutylene terephthalate (PBT) give a component-scale measure of performance.

Secondly, the validated friction model is used in an elastomeric seal model in-situ within the valve and in contact with a polymeric stem surface and subject to both global fittment deformation and canister pressure.

\section{Metered dose inhaler and its valve design and principles of operation}

Several types of device are used to deliver a metered dose of aerosolised medication to the respiratory tract. pMDIs refer to those devices that incorporate a propellant under pressure to generate a metered dose of an aerosol through an atomisation nozzle. These devices consist of several components as shown in Figure 1. The active substance formulated with a propellant and excipients are contained in a canister. A metering valve is crimped onto the canister with an actuator that connects the metering valve to an atomisation nozzle and a mouth piece. The metered volume is typically between 20 to $100 \mu$ l. The metered volume is rapidly expelled from the valve through the actuator orifice where atomisation occurs [7].

The key stages in the drug delivery process of standard valves include filling of the metering chamber, storage and delivery of the dose. During these processes a combination of seals are used to open and close the channels that allow the fluid to freely flow. In Figure 2 two extreme cases are presented. On the left, the valve is at rest; whilst on the right, the stem is fully depressed and the drug is released to the patient. For the valve depicted in Figure 2 the drug delivery process is as follows: 
1. The stem component is in the rest position. The metering chamber contains a metered volume of the formulation.

2. The stem is depressed slowly, closing the channel linking the metering chamber to the bulk.

3. The stem is depressed further, opening the channel in the upper stem and allowing the formulation to flow towards the actuator nozzle.

4. The depressed valve is released, closing the metering chamber to the actuator nozzle.

5. The depressed valve is released further, opening the metering chamber to the bulk formulation in the canister, thus allowing the valve to pre-meter the required next dose.

During these phases the seals deform and slide in relation to other valve components, controlling the metering volume and the dynamic performance of the system.

\section{The friction model}

As an initial study a one-dimensional contact between the deformed seal face-width and the sliding stem is considered. Such an analysis considers the contact behaviour per unit length of the seal contact, when subjected to a sliding motion and fluidic pressure loading. It is, therefore, an approximation, which has also been used by other investigators, dealing with tribology of seals such as Hooke et al [8], Karaszkiewicz [9] and Nikas [10] for seals and orings. However, it should be noted that whilst this is a reasonable simplifying assumption, yielding an analytic solution, the contact geometry is in fact at least partially comforming, requiring a 2-D numerical solution. The resulting 1-D analytical model is then used to obtain 
an estimate of friction due to adhesion, viscous action of a thin film of formulation, as well as any asperity ploughing action.

The values of the contact pressure between the stem and seal during HFA release are used in the friction model, based on the works of Bhushan [11], Bowden and Tabor [12] and Gohar and Rahnejat [13]. The proposed model is appropriate for lightly loaded contacts. This initial study assumes that the fluid viscous behaviour remains Newtonian. However, more details are included for asperity interactions. The total friction force is, therefore, contributed by three phenomena:

$$
F=F_{a}+F_{v}+F_{d}
$$

where $F_{a}$ represents adhesive friction which is the effort required to break the cold-welded junctions between the asperity pairs on the contiguous surfaces. The adhesive friction is obtained as:

$$
F_{a}=A \psi \tau_{s}
$$

where the value of $\psi$ corresponds to the proportion of the contact in direct surface interactions. $A$ is the real contact area, rather than the apparent one, and is given by Bhushan [11] and Gohar and Rahnejat [13] as: 
222 The second term on the right hand side of (1) is due to viscous friction, where:

$223 \quad F_{v}=A(1-\psi) \frac{\eta u}{h}$

224 The third term; $F_{d}$ is the ploughing or deformation friction described later.

225

226 Assuming iso-viscous conditions and noting that the speed of entraining motion of any fluid 227 film, $u$ into the conjunction is half the sliding velocity of the seal, one needs to obtain the film thickness $h$ in order to evaluate $F_{v}$. This is described in section 4.

229

230 Now returning to the third component of friction, $F_{d}$ in equation (1), this is due to the oblique contact of asperity pairs, where those on the harder counterface (in this case on the stem) plough through those on the softer material (the elastomeric seal). This ploughing action may result in elastic or plastic deformation of the softer asperities. Here elastic 234 ploughing of rubber seal asperities is assumed to occur. Thus, according to Gohar and 235 Rahnejat [13]:

236

237

$F_{d}=\pi N f_{d}\left(1-e^{-\Phi V^{\frac{4}{3}}}\right)$

In this study elastic ploughing of asperities is considered, thus:

$239 f_{d}=\pi\left(8 R^{\prime} z_{0}\right) E\left(\frac{R_{q}{ }^{\text {comp }}}{r_{a}}\right)^{\frac{1}{2}}$

And $\Phi$ for the elastic case is given as: 
$\Phi=\frac{1}{8 R^{\prime}}\left\{\frac{\beta m}{2 \pi E}\left(\frac{r_{a}}{R_{q}{ }^{\text {comp }}}\right)^{\frac{1}{2}}\right\}^{\frac{2}{3}}$

The accuracy of predictions partly depends on the validity of the assumption concerning elastic ploughing of asperities and partly on the proportion of kinetic energy expended in asperity deformation, $\beta$, which is assumed to be $\beta=0.8$ in this study [14].

\section{Determination of film thickness in the contact conjunction}

Figure 3 shows the cross-section of a seal (for simplicity considered to be circular (a), squeezed in its retaining groove (b) and under metered chamber pressure (c). Following the simplified analytical approach of Karaszkiewicz [9] a Hertzian contact may be assumed in the fittment of the seal, where the length of the contact is given as $\pi\left(D_{s}+b\right)$, which is large compared with $b$. Also, the effective modulus for the contact $E=E_{r u b}$, since: $E_{\text {stem }}>E_{r u b}$. Thus, using Hertzian theory, Karaszkiewicz [9] showed that the average transverse contact pressure for the assumed infinite line contact condition becomes (see Figure 3(b)):

$$
p_{\text {cont }}=\frac{\pi}{6}\left(\frac{2 a}{b}\right) E_{r u b}
$$

For an analytic solution it is necessary to determine the contact load as the result of this mean pressure, which requires evaluation of the Hertzian contact width 2a. Karaszkiewicz [9] measured the width $2 a$ of a seal squeezed between a glass and a steel plate and obtained an empirical relationship for the ratio $\frac{2 a}{b}$, which agrees well with the finite element results of 
263 George et al [16]. Thus, the mean pressure in equation (8) can be obtained. His empirical 264 relationship is used here as:

265

$266 \frac{2 a}{b}=2 \varepsilon+0.13$

267

268 where $\varepsilon$ is the squeeze ratio described below.

269

270

When the seal is subjected to the canister pressure $p$, the contact pressure distribution alters

as shown in Figure 3 (c), pushing the seal against the groove wall. As the rubber seal is

272 considered to be incompressible $\left(v_{r u b} \approx 0.5\right)$, the contact width alters. A series of experiments carried out by Johannesson [17] suggest that:

$w=\left((2 \varepsilon+0.13)+\left[0.39(1-\varepsilon)^{-1}-0.5(2 \varepsilon+0.13)\right]\left[1-e^{\left(\frac{-4.6 p}{E_{r u b}}\right)}\right]\right) b$

The film thickness required in (3) is estimated from Karaszkiewicz [9] where:

278

279

$h=4.4(\eta u)^{0.65}\left(R_{c}\right)^{0.56}(W)^{-0.21}(E)^{-0.44}$

$W$, the total applied load on the seal is due to a seal fitting into its groove with the fluid 282 pressure load acting behind it: 
$284 W=w\left\{\left(\frac{\pi}{6}\right)(2 \varepsilon+0.13) E_{r u b}+\frac{v_{r u b}}{1-v_{r u b}} p\right\}$

285 where $\varepsilon$ is the squeeze ratio given as: $\varepsilon=\frac{b-g}{b}$. In the present case it is $\varepsilon=0.102$.

$w$ is the effective width of the contact that the seal makes with the stem, when fitted in-situ and subjected to a pressure, $p$.

\section{Materials used and methods of measurement}

Nitrile rubber and PBT samples were used for friction tests described below. All experiments were performed under ambient conditions $\left(40 \pm 1 \% \mathrm{RH}\right.$, temperature $\left.20 \pm 0.5^{\circ} \mathrm{C}\right)$.

The simulations were carried out assuming that the physical properties of the liquid contained in the metered chamber are those of pure HFA 227a. The presence of surfactant and drug in the mixture was neglected due to their low concentrations.

\subsection{AFM imaging and nano-scale friction force acquisition}

An atomic force microscope (Nanoscope IV, Digital Instruments) was used to initially characterise the surface topography of counterfaces. This data is required for the adhesive and ploughing components of the developed friction model. The AFM is also used to determine the coefficient of friction.

Roughness measurements were carried out in the tapping mode, while friction measurements were conducted in lateral force mode. V-shaped micro-fabricated $(100 \mu \mathrm{m})$ cantilevers with 
pyramidal, oxide-sharpened $\mathrm{Si}_{3} \mathrm{~N}_{4}$ tips, supplied by Digital Instruments (model DNP, spring constant of $0.58 \mathrm{~N} / \mathrm{m}$ ) were used for all the friction measurements. Surface friction force data was acquired by simultaneously scanning in the forward $(+x)$ and reverse $(-x)$ directions with disabled scanning in the $y$ direction. The sliding tip velocity was set at $50 \mu \mathrm{m} / \mathrm{s}$ with the scan frequency of $1 \mathrm{~Hz}$. Each measurement used here represents an average of at least five independent scans.

The raw friction data in volts output was determined from half the difference between the retrace (right-to-left) and trace (left-to-right) 512- by 512-pixel lateral force images. The friction force image with subtraction is shown in Figure 4. All the measured friction data sets were fitted with Gaussian distribution in order to obtain mean values and standard deviations.

The static friction coefficient between rubber and $\mathrm{Si}_{3} \mathrm{~N}_{4}$ tip was determined by measuring the maximum value of the lateral deflection of the AFM tip [18].

To convert the raw friction data (in volts) into lateral forces (in Newton) a lateral force calibration factor $\alpha$ (in $\mathrm{V} / \mathrm{nN}$ ) was obtained according to the calibration procedure described in Ahimou et al [19]. Silicon wafers were used as the calibration standard. The silicon wafers were cleaned for $10 \mathrm{~min}$ in acetone, rinsed with deionised water and dried by adding a few drops of ethanol to remove excess water. Measurements were performed before and after each rubber test to ensure that the state of the AFM probe remained unaltered. A step increase in applied load between $0-200 \mathrm{nN}$ was employed per image from a $100 \mu \mathrm{m}^{2}$ region of silicon wafer surface (Figure 5). The scan velocity was $50 \mu \mathrm{m} / \mathrm{s}$ at $0.5 \mathrm{~Hz}$ scan frequency. In each case, the plot of raw friction force in volts versus the applied load in $\mathrm{nN}$ was reproduced by a linear fit, consistent with Amontons' law of friction [20] with the slope $\mathrm{k}_{\mathrm{SiOx}}$ determined in 
units of $\mathrm{V} / \mathrm{nN}$. This slope is equal to the "to- be-determined" apparatus coefficient times the actual friction coefficient obtained by $\mu_{\text {SiOx }}=0.19 \pm 0.01$, averaged from the data obtained by Buenvuaje et al [21] and Putman et al [22]. All the raw friction force values (volts) measured during friction tests for rubber were divided by the value for $\alpha(\mathrm{V} / \mathrm{nN})=\mathrm{k}_{\mathrm{SiOx}} / 0.19$ to convert them to calibrated friction force levels in units of $\mathrm{nN}$. The friction coefficient was determined dividing the measured nano-scale friction force by the applied load of 50nN.

\subsection{Tribometric device}

Component-level (macro-scale) friction between the nitrile rubber and a PBT flat sheet was measured using a traditional friction test apparatus; an instrumented trolley test. A schematic of the device is shown in Figure 6. The force transducer measures the friction between the contacting surfaces, whilst the sliding velocity is recorded by a laser vibrometer (Polytec model 302), shun on the sliding trolley surface.

The raw friction data (volts) and sliding velocity (volts) were recorded in real time. Knowing the sensitivity $(25 \mathrm{~mm} / \mathrm{s} / \mathrm{V})$ of the vibrometer, the sliding velocity was obtained. The measured sliding velocity during the experiments was up to $0.06 \mathrm{~m} / \mathrm{s}$. The calibration procedure was adopted to convert the acquired friction signals from Volts to Newtons. Before experiments a load cell (capacity: $0.3-3 \mathrm{~kg}$ ) was calibrated with known weights to obtain measurement friction sensitivity. All raw friction force values (in Volts) were multiplied by the friction sensitivity $(1.18 \mathrm{~N} / \mathrm{V})$ to convert them to friction force. The friction coefficient was obtained by dividing the friction force by the applied normal load (4.51 N). 


\subsection{Statistical analysis}

To determine whether the average of the measurements of $R q$ roughness values for a sample of ten gaskets was statistically sufficient to represent this parameter; the following procedure was followed [23].

The RSS (residual sum of squares) was estimated for the ten $R q$ values and for each of the combinations with 9 values. Then the value of " $p$ " was determined as:

$f=\frac{\left(R S S_{2}-R S S_{1}\right) /\left(D F_{1}-D F_{2}\right)}{R S S_{1} / D F_{1}}$

where $D F$ is the degree of freedom (number of $R q$ values for average determination - 1) and the subscript 1 refers to the ten samples and 2 to each of the combinations of nine samples.

The value of $f$ was compared with the values from the $F$ distribution with $D F_{1}-D F_{2}$ and $D F_{1}$ values of freedom $\left(F_{D F_{1}}^{D F_{2}-D F_{2}}\right)$ at a set probability (for this work it was set at 0.05 ); if $f>$ $F_{D F_{1}}^{D F_{2}-D F_{1}}$ then the addition of the tenth $R q$ value is of benefit, otherwise the difference between $R S S_{1}$ and $R S S_{2}$ is smaller than the measuring error $\left(R S S_{1}\right)$.

The average values of surface roughness $R q$ determined in three points along the face-width of the seal (Figure 7) were compared with the one way ANOVA test followed post hoc by the Tukey's test for individual pairs ( $p$-value $<0.05)$. These analyses were performed using the SPSS software.

\section{Results and discussion}

\subsection{Determination of parameters for the friction model}

Input data for the friction model (section 3) requires measurement of surface roughness parameters such as those for rubber and PBT, $R_{q}$, and the RMS composite surface 
roughness, $R_{q}^{\text {comp }}$, average tip radius of asperities, $r_{a}$, as well as determining a representative value for radius of hemispherical asperities, $R^{\prime}$. It is also necessary to determine the number of asperities in the real contact area, $N$.

Surface roughness $(R q)$ for rubber and PBT samples were obtained from samples of 10 by 10 $\mu \mathrm{m}$ AFM images. An example of an AFM image for the nitrile rubber is shown in Figure 8. A statistical F-test revealed that the average value of $R q$ obtained from nine measurements was adequate to describe this parameter.

The value of $R q$ was also estimated in different locations along the seal facewidth to determine if the manufacturing method makes any significant differences. It was found that the values of roughness were statistically different $(p-$ value $<0.05)$. Moreover the Tukey's test showed that the roughness in position A was different from that in locations B and C. The normalised frequency of $R q$ at the three locations chosen is shown in Figure 9. It can be seen that the average value of $R q$ in $\mathrm{B}(R q=1.04)$ and $\mathrm{C}(R q=1.13)$ are very close, whilst that at position $\mathrm{A}$, it was $(R q=1.51)$, the standard deviation of $R q$ at $\mathrm{B}$ is smaller $(0.12)$ than at $\mathrm{A}$ and C (0.28 and 0.30 respectively). As there are two statistically different values for $R q$, the friction model simulations were carried out with both the values of $R q$ at positions $\mathrm{A}$ and $\mathrm{B}$ (see Table 1).

The composite surface roughness was calculated as $R_{q}^{\text {comp }}=\sqrt{R_{q_{-} r u b}^{2}+R_{q_{-} \text {stem }}^{2}}$, where $R_{q_{-} \text {rub }}$ and $R_{q_{-} \text {stem }}$ are the surface roughness values for rubber and PBT stem respectively. 
405 Other surface parameters required for the friction model are the average asperity tip radius

$409 \quad R^{\prime}=\frac{l^{D_{f}}}{G^{\left(D_{f}-1\right)}}$ and height. The asperities were assumed to be hemispherical in shape with a radius $R^{\prime}$ and a base diameter $l$, such that the base area is proportional to $l^{2}$. The radius of curvature, $R^{\prime}$, for the asperity can be found as (Bhushan [11]):

The fractal dimension, $D_{f}$, of the roughness profile is then calculated using two methods: (i)enclosing boxes and (ii)- morphological envelopes. The average value of fractal dimension obtained from these two methods is used to calculate the radius of curvature and the height of an asperity.

Also the average typical radius of the asperity was determined independently. A surface $(10 \mathrm{x}$ $10 \mu \mathrm{m}$ ) was scanned along lines spaced by intervals of $0.25 \mu \mathrm{m}$. For each line the $\mathrm{z}-$ coordinate of the surface was measured. Along each line the peaks (local maxima) were identified as points, whose z- coordinate was higher than the coordinates of three consecutive points prior to and after it. This procedure led to the estimation of the total number of peaks in the scanned area (number of peaks $/ \mu \mathrm{m}^{2}$ ). This value was later used to estimate the total number of peaks presented in the real area of contact. The coordinates of these seven points were interpolated with a parabolic equation (the values of the three parameters were estimated according to the minimal residual sum of square methods with an in-house algorithm running in Excel 2003).

For each peak the curvature radius was determined from the fitting equation using the following expression: 
$r_{a}=\frac{\left[1+\left(\frac{\partial z}{\partial x}\right)^{2}\right]^{3 / 2}}{\frac{\partial^{2} z}{\partial x^{2}}}$

431 Finally, the average value and standard deviation of the curvature radius of the surface peaks 432 was determined.

433

434 The number of asperities was determined both experimentally (see above) and numerically.

435 The numerical procedure was based on expressions for circular contact footprints described 436 by Gohar and Rahnejat [13]. This assumes that both surfaces are nominally flat, but one of 437 them has isotropic roughness features with identical spherically shaped asperities on it.

438

439 The normal load on each asperity is defined as:

$440 \quad W_{i}=\frac{4}{3}\left(E R^{\prime 1 / 2} \delta_{i}^{3 / 2}\right)$

441 and the contact centre deflection for each asperity according to the classical Hertzian theory 442 is:

$443 \quad \delta_{i}=\left(\frac{9 W_{i}^{2}}{16 E^{2} R^{\prime}}\right)^{1 / 3}$

444 The contact area for one elastic spherical asperity in terms of its deflection is then defined as:

$445 \quad A_{i}=\pi R^{\prime} \delta$

446 An iteration procedure is adopted to determine the contact area for one asperity.

447 Knowing $A_{i}$ the total number of asperities can then be found as: 
$448 \quad N=\frac{A}{A_{i}}$

454

The numerically obtained number of asperities agrees reasonably with the experimentally extracted values (Table 1). The experimentally obtained surface roughness parameters used in the development of the friction model are also summarised in Table 1.

\subsection{Validation of the friction model}

The friction model was validated against experimental results performed at both nano and component level (macro) scales. The friction on nano-scale was measured using AFM while a sliding trolley test rig was used for obtaining friction data on the macro-scale. The experiments were carried out under dry conditions, consequently, there was no viscous force involved due to the absence of the lubricant film.

Experimentally obtained friction results are presented in Table 2. Friction model predictions give the value of coefficient of friction as 0.69 for the nitrile rubber-PBT combination. The same model applied for the nano-scale friction returns a coefficient of friction of 0.17 , when the input parameters are those for the nitrile rubber and the silicon nitride AFM tip. Therefore, the predictions for the coefficient of friction for the nano-scale conforms reasonably well to the measured values, with an average percentage error of around $14 \%$, while for the component-level (macro-scale) scale the predictions give an error of around 23\%. The effect of different surface roughness parameters of position A and B was to have negligible effect on the outcome of the model simulations. 


\subsection{Friction results for the pMDI valve}

473

474

The validated friction model is then used for the pMDI valve operation. During the stem movement the pressure decreases from the initial 3.9 bar to atmospheric. The calculations of friction forces, their coefficients and the lubricant film thickness have been performed at several pressures within this range at a fixed sliding velocity of $20 \mathrm{~mm} / \mathrm{min}$. This velocity was chosen as it is the typical sliding velocity in the pMDI valve. The static friction coefficient is of main interest in this work, since the highest friction is observed during start up of the inhaler mechanism.

The total friction force (Figure 10) increases with pressure in the metering chamber due to an increase in the contact area with applied load. Therefore, the coefficient of friction remains almost constant at a value of 0.69 through pressure changes (Figure 11). The film thickness variation is negligible with increase of pressure in the metering chamber and stays in the range of a fraction of a nanometer $(0.21-0.22 \mathrm{~nm})$. This explains the insensitivity of friction variation with sliding velocity, indicating dominance of adhesive component of friction.

The total friction coefficient and its friction force are the sum of adhesive, viscous and ploughing terms contributions. The adhesive friction is dominant. Viscous and ploughing contributions are found to be insignificant. In fact, with pressure increases in the metered chamber the viscous friction force varies from 5.97 to $4.20 \mu \mathrm{N}$, while the ploughing friction force changes from 15.5 to $11.6 \mu \mathrm{N}$. The viscous friction is negligible because no film is in effect formed and the working sliding velocity is also very low $(20 \mathrm{~mm} / \mathrm{min})$. This low sliding velocity, together with the rather smooth surface roughness profile of the contiguous surfaces makes the ploughing contribution also insignificant. Ploughing is as a result of hard, 
mostly conical shape asperities deforming their counterparts on the softer material.. When, as in this case, the asperity angle is large the ploughing component of friction is correspondingly insignificant. Thus, the calculated points in figure 11 are really due to adhesive friction.

\section{Conclusions}

The friction model has been developed and validated on both nano and component level (macro) scales. Results show that the adhesive friction is dominant. Contributions of viscous and ploughing frictions are minor. To improve frictional behaviour, edge profiling of the seal may be undertaken in order to encourage lubricant entrainment into the contact by wedge effect (see Nikas [24]). However, seal edge-profiling can cause loss of effective sealing and detailed numerical analysis would be required, which points to one aspect of future work. The edge profiles also require manufacturing control, which may become cost ineffective.

The effort required to actuate the valve is a very important performance parameter because of the wide range of possible users with different strength; the measurement can be achieved through the hysteresis cycle (see Grimble et al [5]). This work can be used to predict the frictional behaviour of pMDIs and form the basis for their further development. 


\section{Acknowledgements} technical support extended to this investigation.

9. References

522

[1] Everard M.L., CFC transition: the Emperor's new clothes. Each class of drug deserves a delivery system that meets its own requirements, Thorax 55, 2000, pp. 811-819.

[2] Schultz R, Dupont R, Ledoux K., Issues surrounding metered dose valve technology: past, present and future perspectives, Proceedings of the Respiratory Drug Delivery Conference - IV, 1994. Richmond: Interpharm Press, 1994, pp.211-220.

[3] FDA, Guidance for industry metered dose inhaler (MDI) and dry powder inhaler (DPI) drug products, CDERGUID/2180dft.wpd. Nov. 1998, http://www.fda.gov/cder/guidance/2180dft.pdf, 1998.

[4] Howlett D, Colwell J, Goldsmith S, McCallion O., Correlation of extractables and leachables from marketed pMDIs, Proceedings of the Respiratory Drug Delivery Conference - VIII, 2002. Richmond: Virginia Commonwealth University, 2002, pp. 129-136.

[5] Grimble DW, Theodossiades S, Rahnejat H. and Wilby M., Tribology of rough ultrafilm contacts in drug delivery devices. Proc IMechE Part C: J. Mechanical Engineering Science 2008, 222, pp. 2209-2216.

[6] Rahnejat H., Multi-body Dynamics: Vehicles, Machines and Mechanisms, Professional Engineering Publishing and SAE (joint Publishers), London and Warrendale, $\mathrm{Pa}, 1998$. 
[7] Clark A., MDIs: physics of aerosol formulation, J Aerosol Med 1996; 9: S19-S26.

[8] Hooke G.J, Lines D.J, O’Donoghue J.P., Elastohydrodynamic Lubrication of O-ring Seal, Proc Inst Mech Eng, 1966, 181, pp. 205-210.

[9] Karaszkiewicz A., Hydrodynamics of rubber seals for reciprocating motion, lubricating film thickness, and out-leakage of O-seals, Ind Eng Chem Res, 1987, 26, pp. $2180-2185$

[10] Nikas G.K., Transient Elastohydrodynamic Lubrication of Rectangular Elastomeric Seals for Linear Hydraulic Actuators, Proc Instn Mech Engrs; J Engng Trib., 2003, 217, pp. 461-473.

[11] Bhushan B, Principles and Applications of Tribology, New York: John Wiley and Sons, 1999.

[12] Bowden F.P. and Tabor, D., The Friction and Lubrication of Solids, Oxford: Clarendon Press, 2001.

[13] Gohar R. and Rahnejat H., Fundamentals of Tribology, London: Imperial College Press, 2008.

[14] Sabey BE., Pressure Distributions beneath Spherical and Conical Shapes pressed into a Rubber Plane, and their Bearing on Coefficients of Friction under Wet Conditions, Proc Phys Soc., 1958, 71(B), pp. 979-980.

[15] Karaszkiewicz A., Geometry and Contact Pressure of an O-Ring Mounted in a Seal Groove, Ind Eng Chem Res, 1990, 29, pp. 2134-2137.

[16] George A.F, Strozzi A. and Rich J.I., Stress fields in a compressed unconstrained elastomeric O-ring seal and comparison of computer predictions with experimental results, 11th International Conference on Fluid Sealing; BHRA (British Hydromechanics Research Association): Cranfield Bedford, England, 1987, paper B1, pp. 117-137. 
[17] Johannesson H., Calculation of the pressure distribution in an O-ring seal contact, 5th Leeds-Lyon Symposium on Tribology,The University of Leeds, England, 1978.

[18] Sundararajan S. and Bhushan B., Static friction and surface roughness studies of surface micromachined electrostatic micromotors using an atomic force/friction force microscope, J. Vac. Sci. Tech. A, 2001, 19, pp. 1777-1785.

[19] Ahimou F, Semmens M, Novak P. and Haugstad G., Biofilm cohesiveness measurement using a novel atomic force microscopy methodology, J. Appl. and Environmental Microbiology , 2007, 73(9), pp.2897-2904.

[20] Gao J, Luedtke W, Gourdon D, Ruths M, Israelachvili J. and Landman U., Frictional forces and Amonton's law: from the molecular to the macroscopic scale, $J$ Phys Chem B, 2004, 108, pp. 3410-3425.

[21] Buenvuaje C, Ge S.R., Rafailovich M, Overney R., Atomic force microscopy calibration methods for lateral force, elasticity and viscosity, Mater. Res. Soc. Symp. Proc., 1998, 522, pp.187-192.

[22] Putman C, Igarashi M. and Kaneko R, Quantitative determination of friction coefficients by friction force microscopy, Jpn J. Appl Phys 1995, 34, L264-L267.

[23] Zwietering $\mathrm{MH}$, Jongenburger I, Rombouts F.M. and Riet K., Modeling of the bacterial growth curve, J. Appl. and Environmental microbiology, 1990, 56(6), pp.1875-1881.

[24] Nikas, G.K., Fundamentals of sealing and tribology of hydraulic reciprocating seals. Focus on reciprocating seals: exploring the latest developments and their applications, IMechE, London, 2008. 


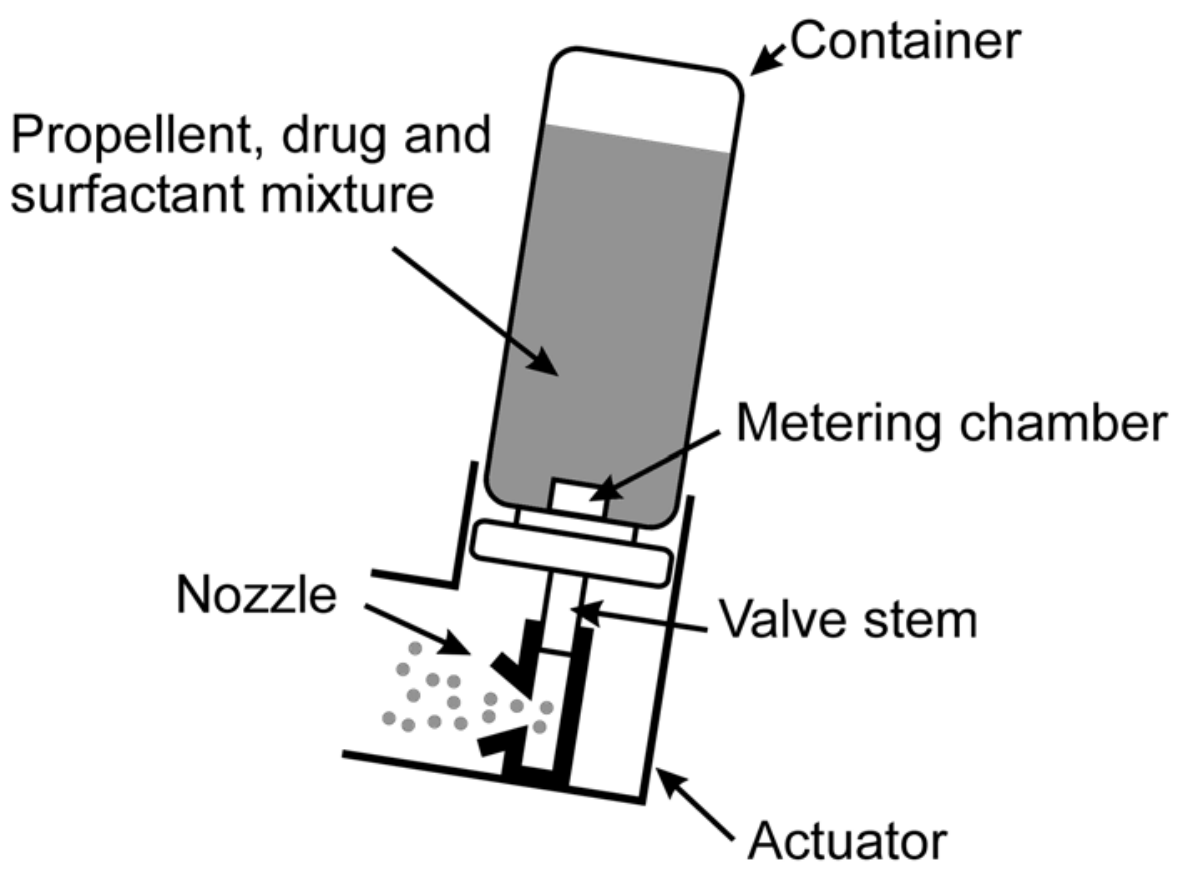

Figure 1: Schematic of a pressurised metered dose inhaler

Figure 2: Schematic of a typical valve configuration: on the left - valve at rest; on the right - stem fully depressed and drug released to recipient. 


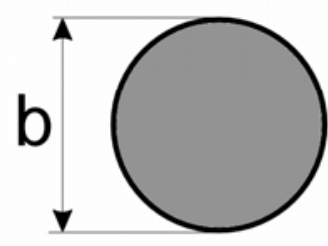

602

603

604

605

606

607

608

609

610

611

612

619

620

621

622

623

624
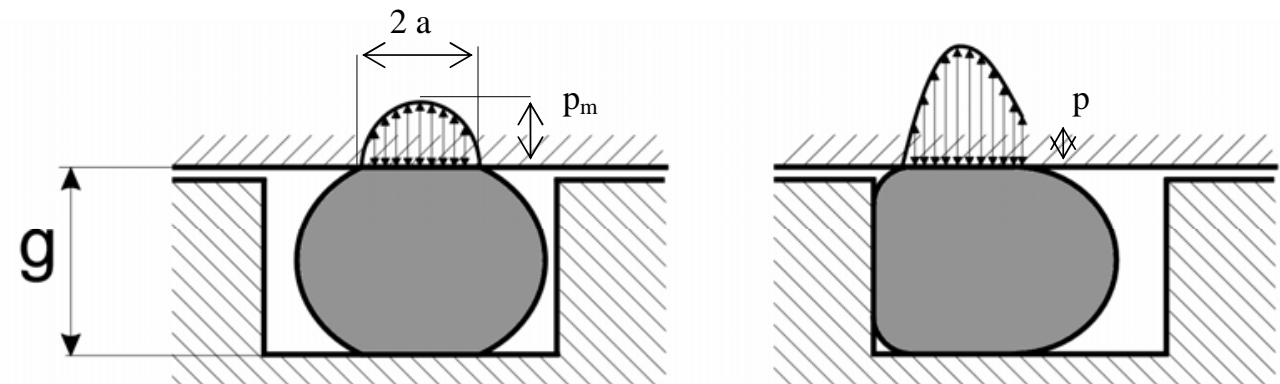

a

b

C

Figure 3: Distribution of contact pressure and geometry of the $\mathbf{O}$-ring mounted in the seal groove and subjected to sealed pressure: (a) underformed seal; (b) deformed insitu due to fitment . (c) at metered chamber pressure of $p$.

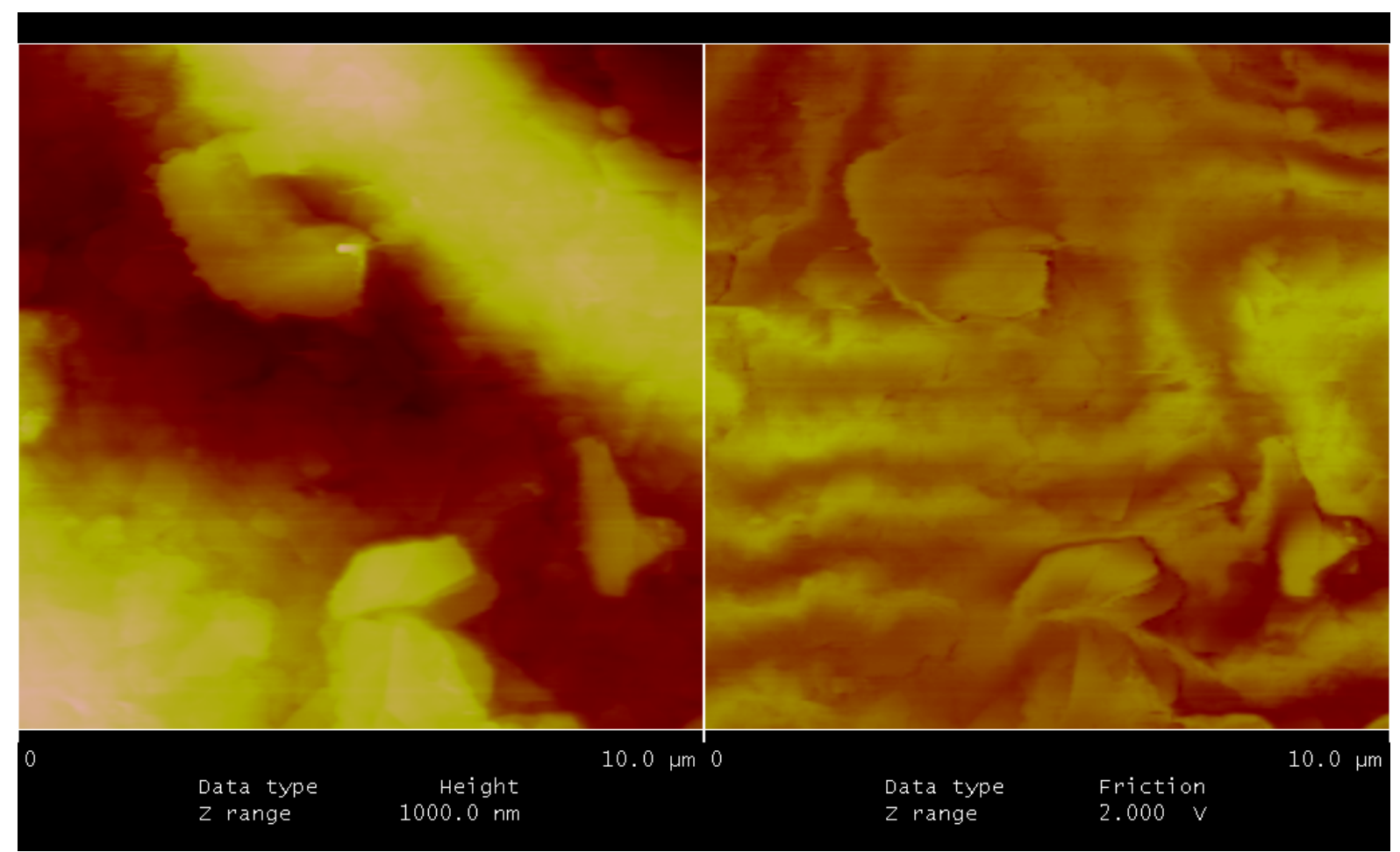

Figure 4: Topographic (on the left) and friction force with subtraction (on the right) images of 10 by $10 \mu \mathrm{m}$ of nitrile rubber 


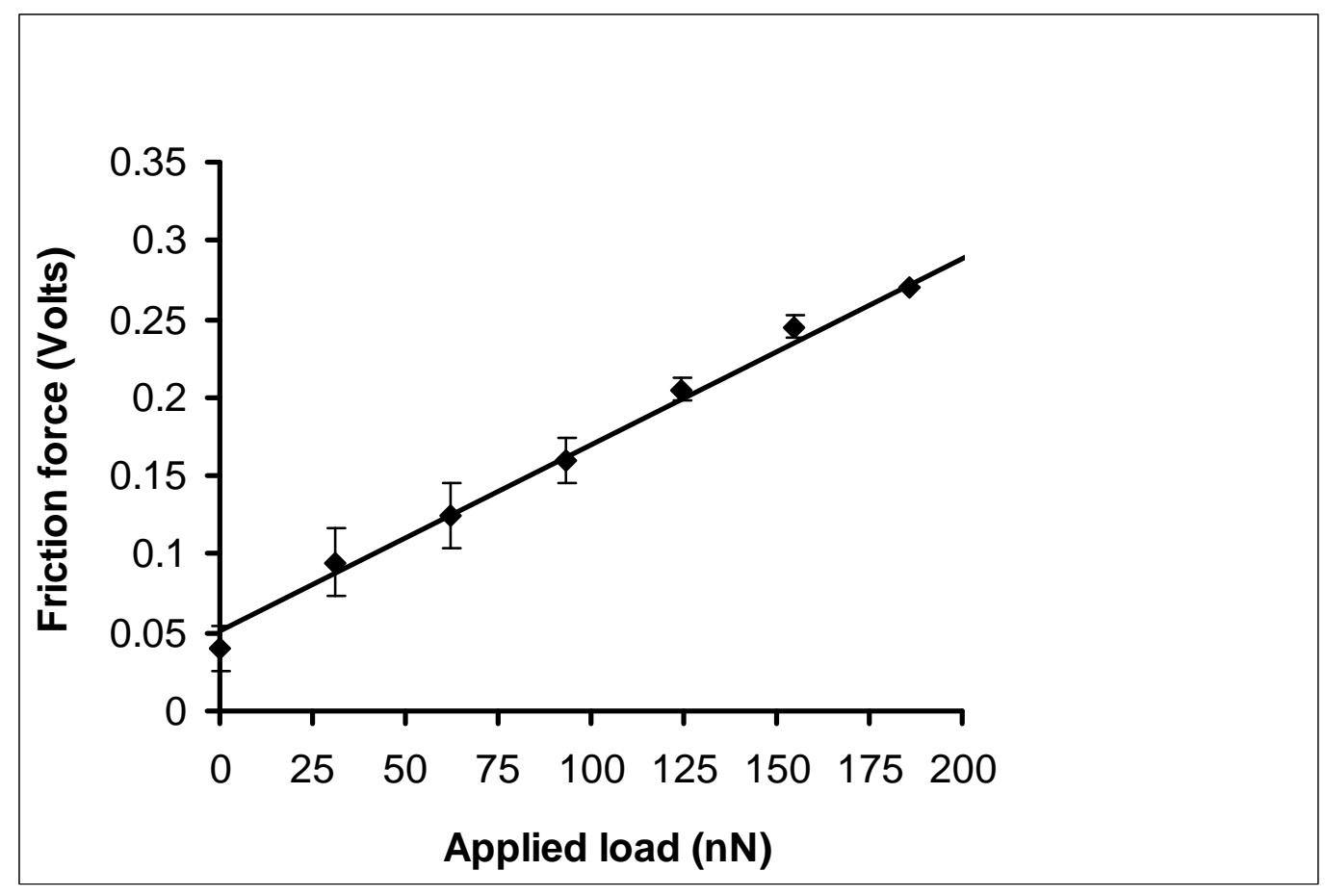

Figure 5: Calibration curve for silicon wafer

628

629

630

631

632

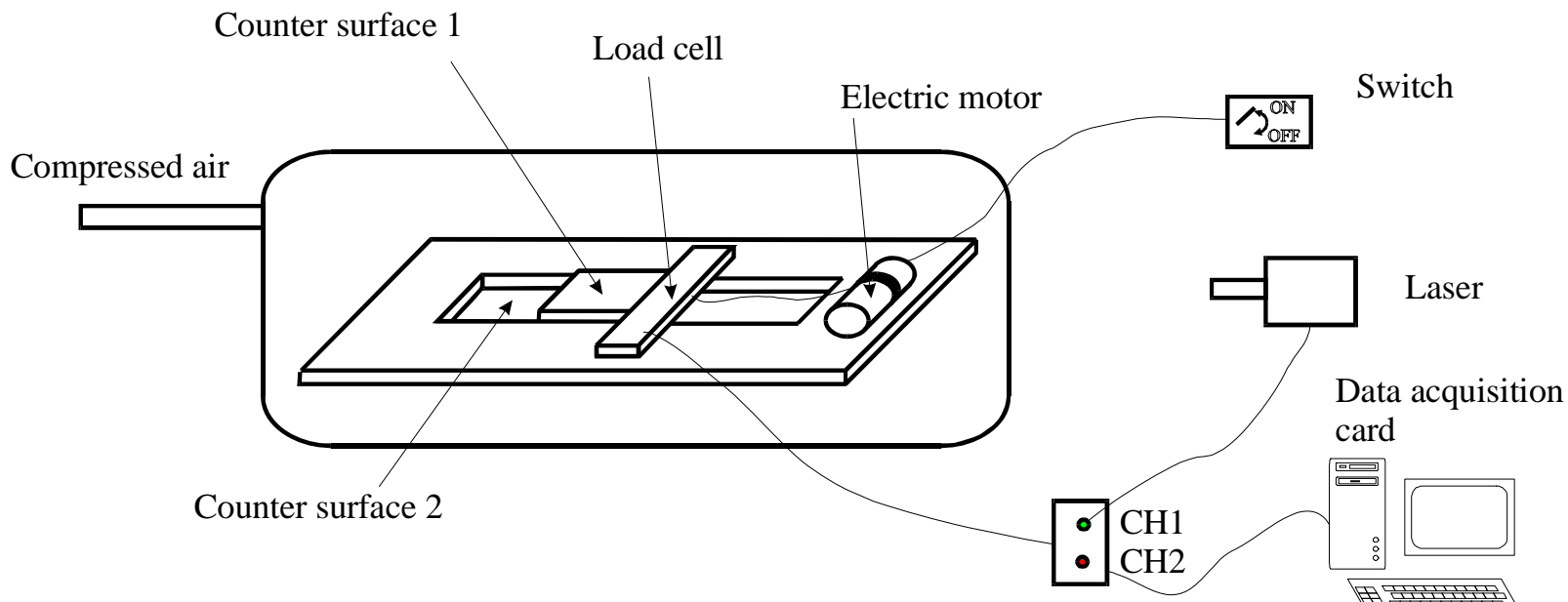

Figure 6: A schematic of the friction test apparatus 


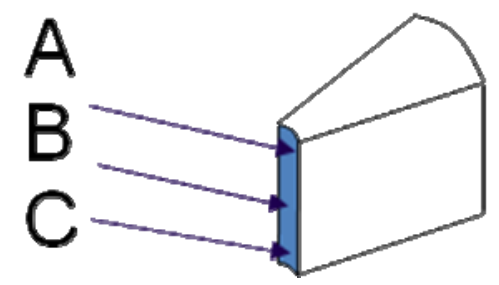

647

Figure 7: Cross section of the rubber seal

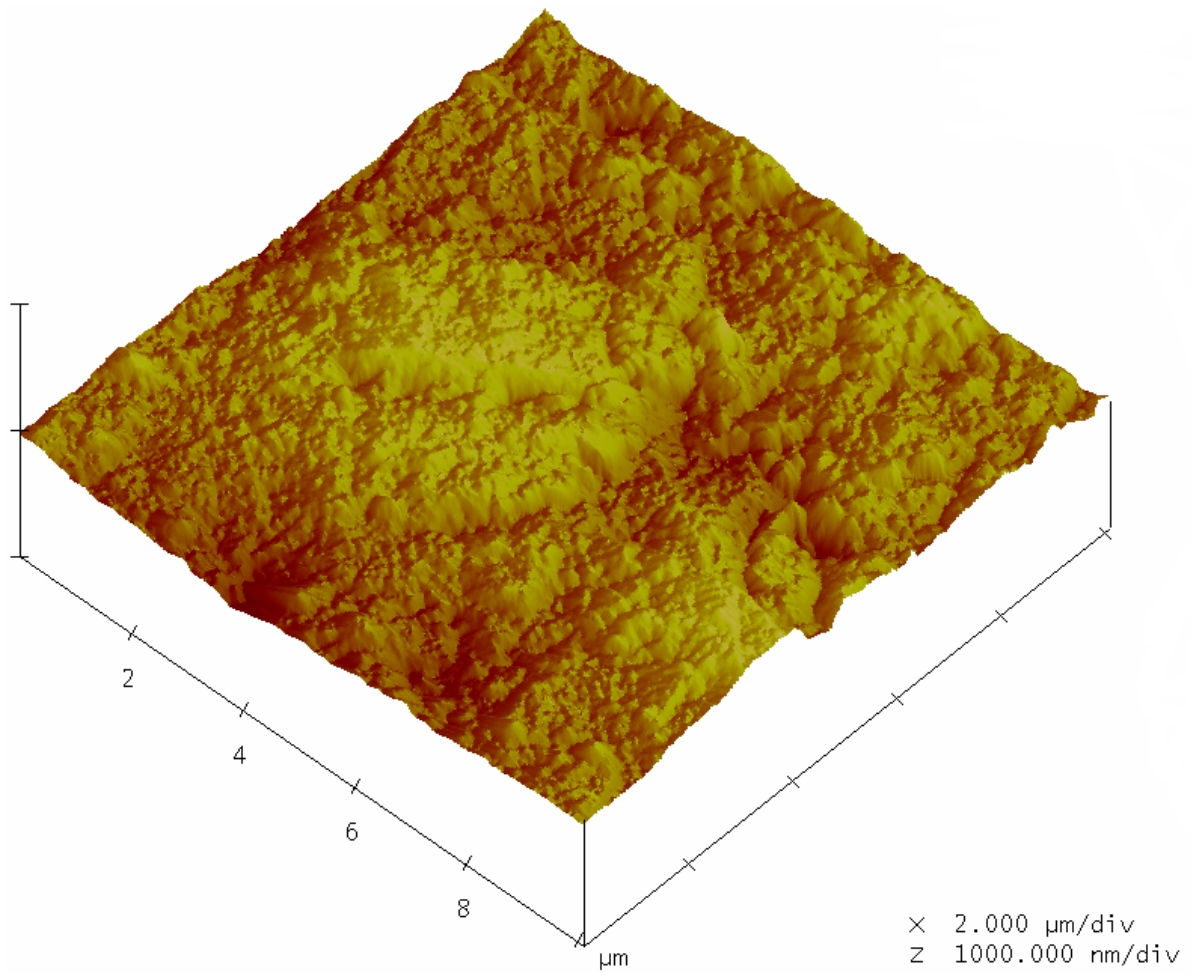

649

650

651

652

Figure 8: An AFM image of the nitrile rubber surface

653 


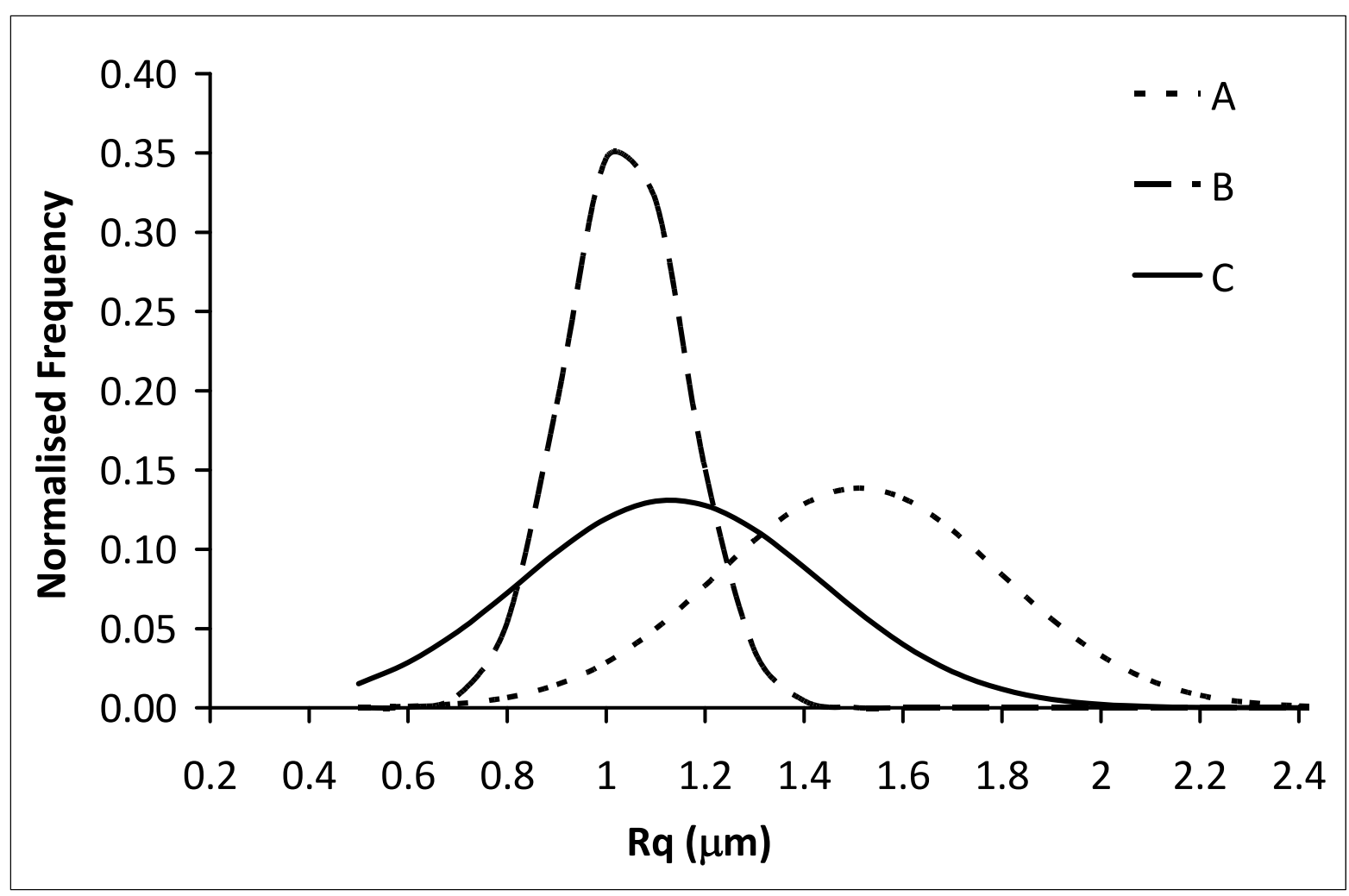

Figure 9: Statistical analysis of $R q$ roughness data for the rubber seals

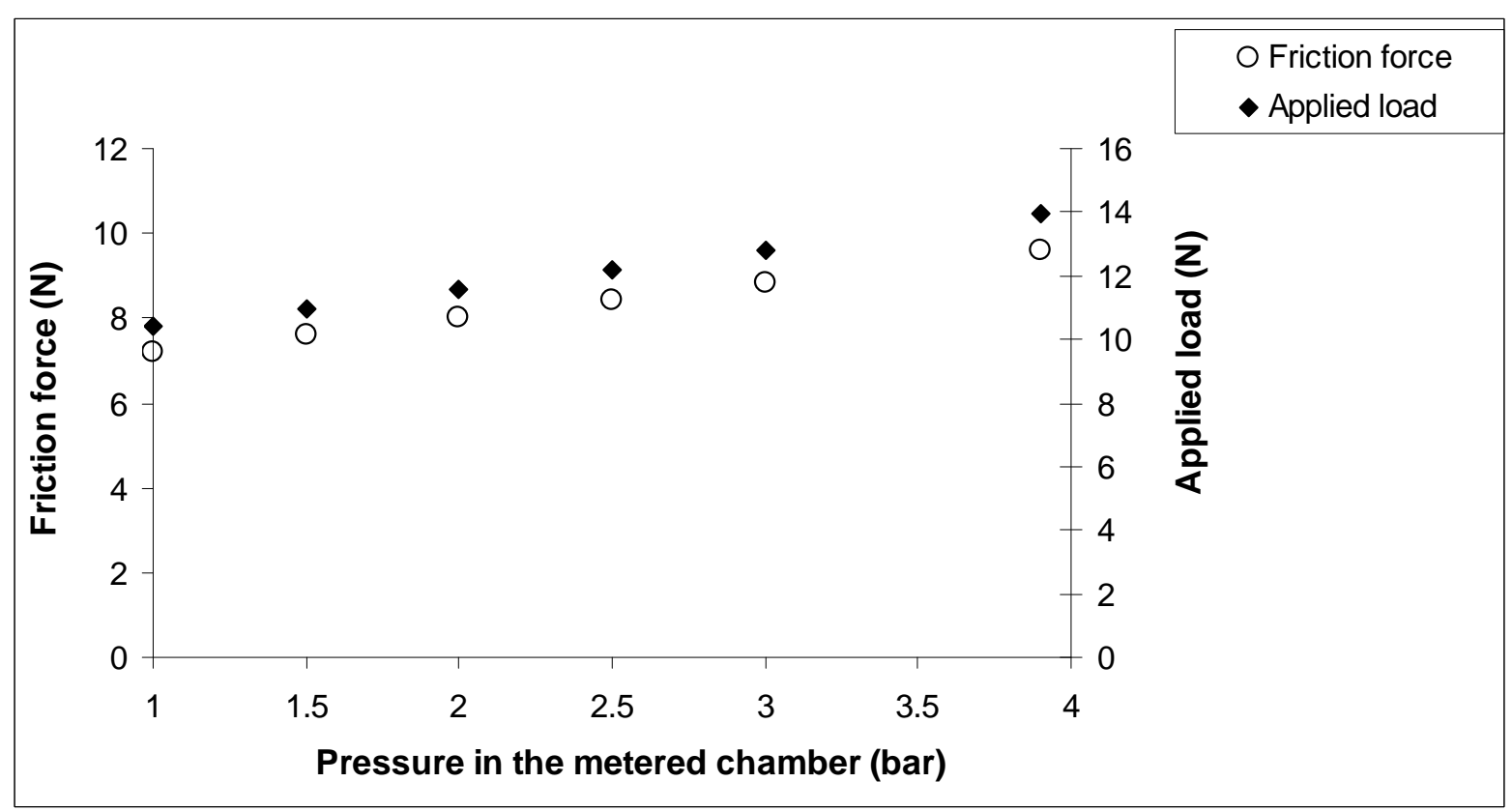

Figure 10: Friction force and applied load variations with chamber pressure 


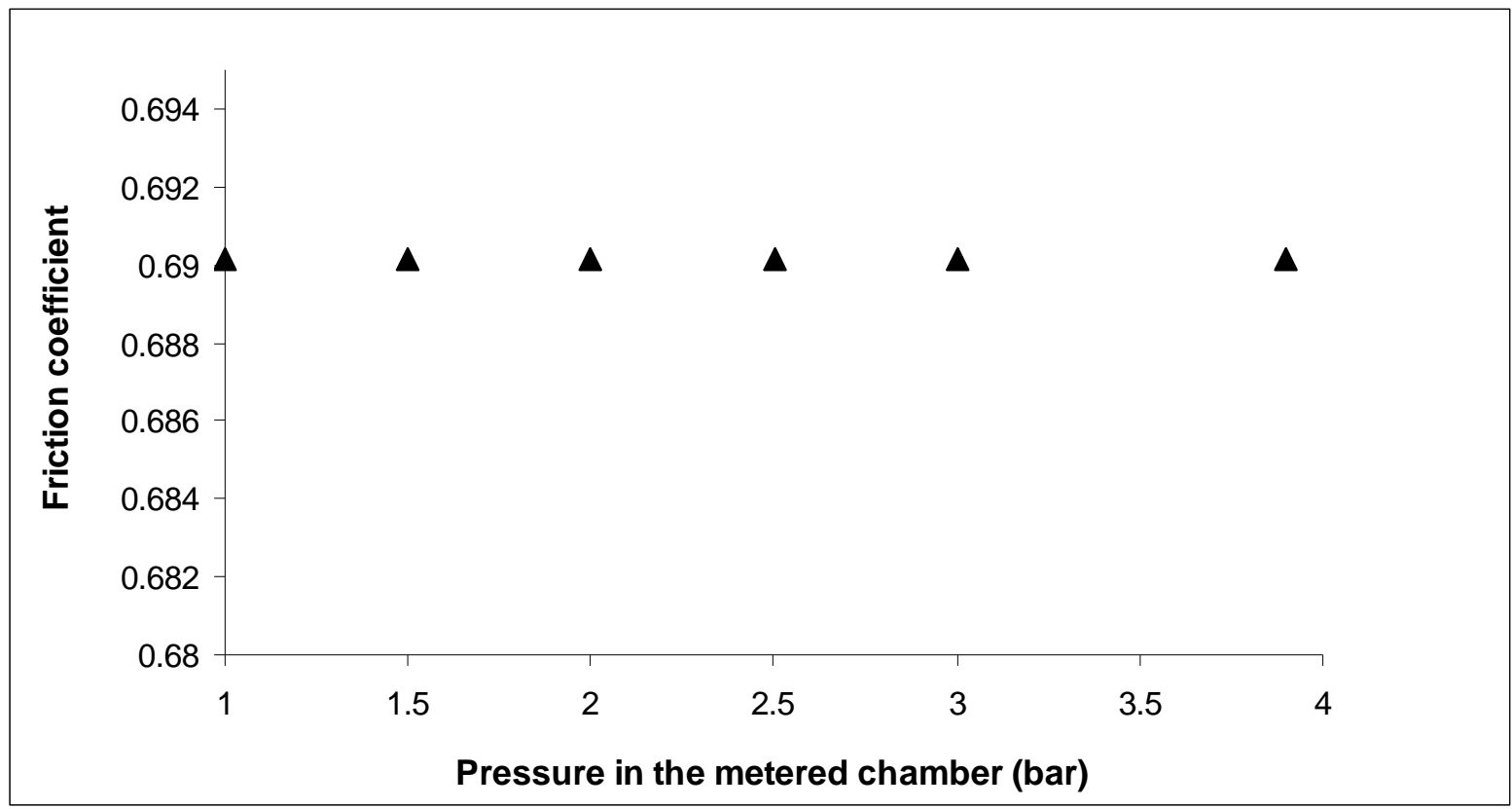

670

Figure 11: Coefficient of friction variation with chamber pressure

672

673

674

675

676

677 
682

689

690

691

692

693

\section{Tables}

Table 1: Experimentally obtained surface roughness parameters

\begin{tabular}{|l|l|l|l|}
\hline $\begin{array}{l}\text { Roughness } \\
\text { parameters }\end{array}$ & $\begin{array}{l}\text { Rubber gasket, } \\
\text { position A }\end{array}$ & $\begin{array}{l}\text { Rubber gasket, } \\
\text { position B }\end{array}$ & PBT \\
\hline$R_{q}(\mu \mathrm{m})$ & $1.51 \pm 0.28$ & $1.04 \pm 0.12$ & $0.31 \pm 0.03$ \\
\hline$R^{\prime}(\mu \mathrm{m})$ & $1.84 \pm 0.09$ & $2.12 \pm 0.08$ & \\
\hline$z_{0}(\mu \mathrm{m})$ & $1.76 \pm 0.04$ & $1.72 \pm 0.05$ & \\
\hline$r_{a}(\mu \mathrm{m})$ & $1.53 \pm 0.04$ & $1.55 \pm 0.07$ & \\
\hline$N($ experimental) & $1.4^{*} 10^{10}$ & $1.4^{*} 10^{10}$ & \\
\hline$N$ (numerical) & $2.5^{*} 10^{10}$ & $2.5^{*} 10^{10}$ & \\
\hline
\end{tabular}

Table 2: Coefficients of friction on nano- and component level scales

\begin{tabular}{|l|l|l|l|l|}
\hline $\begin{array}{l}\text { Friction } \\
\text { coefficient }\end{array}$ & \multicolumn{2}{|c|}{ Nitrile rubber/PBT } & \multicolumn{2}{c|}{ Nitrile rubber/ $\mathrm{Si}_{3} \mathrm{~N}_{4}$ AFM tip } \\
\hline & Experimental & analytical & experimental & analytical \\
\hline & $0.59 \pm 0.03$ & 0.69 & $0.21 \pm 0.025$ & 0.17 \\
\hline
\end{tabular}

